\title{
THEORY OF YARN UNWINDING FROM PACKAGES
}

\author{
PRACEK, S. \& KRAJNER, M.
}

Abstract: In textile production the yarn is being withdrawn from cross-wound packages in warping and weft insertion. During unwinding there appear forces in the yarn that are approximately proportional to the square of the unwinding velocity. The yarn tension is not constant, but it oscillates within some interval. This is especially noticeable in over-end unwinding from a static cross-wound package. Even when the yarn is not strongly stressed, so that the tension does not exceed a few percent of the breaking strength, the yarn still can break sometimes. We strive to achieve as large warping and weaving speeds as possible, therefore our aim is to improve the theory of cross-wound package unwinding and to find the necessary modifications of the yarn unwinding process. We state the equations of motion that describe the unwinding yarn and develop a mathematical model, which permits to simulate the process of unwinding.

Key words: yarn unwinding, equations of motion, balloon theory, simulations, oscillations
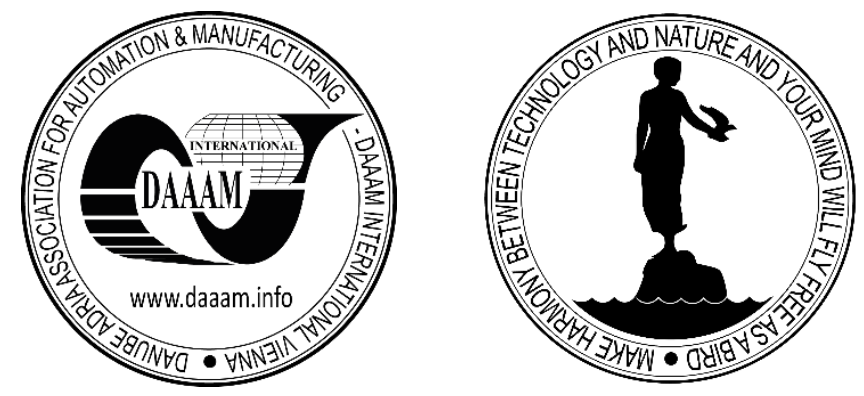

Authors' data: Assoc.Prof. Pracek, S[tanislav]*, Krajner, M[arko]**, * University of ljubljana, NTF, Department of TGO, Snezniska 5, 1000 Ljubljana, Slovenija, ** EPEA Switzerland GmbH, Seestrasse 119, CH-8806 Bäch, Switzerland stane.pracek@ntf.uni-lj.si, krajner@epeaswitzerland.com

This Publication has to be referred as: Pracek, S[tanislav] \& Krajner, M[arko] (2018). Theory of Yarn Unwinding from Packages, Chapter 10 in DAAAM International Scientific Book 2018, pp.107-118, B. Katalinic (Ed.), Published by DAAAM International, ISBN 978-3-902734-19-8, ISSN 1726-9687, Vienna, Austria DOI: $10.2507 /$ daaam.scibook.2018.10 


\section{Introduction}

Yarn unwinding is an essential step in many textile processes such as ring spinning and two-for-one twisting (Barr\&Catling, 1976; Clark et al.,1998). The quality of the fabric that is produced directly depends on the regularity of the unwinding the tension in the yarn should be low and constant.

The characteristics of the unwinding process are thus important for production of high quality garments and should therefore be optimized.

The theory of unwinding and balloon formation originate from the pioneering work of D. Padfield (Padfield, 1956). She modified Mack's equations (Mack, 1953) and included terms that describe the Corolis force.

She found solution for a balloon that forms during unwinding from stationary cylindrical package when quasistationary conditions apply.

This theory was also used for calculations of multiple balloons and for balloons formed during unwinding from packages with different geometry, such as conic packages (Padfield, 1958).

Kothari and Leaf have rederived similar equations, additionally taking into account the effect of gravity and tangential air drag (Kothari\&Leaf, 1979).

Using elaborate numerical calculates they have shown that these two forces have only a small contribution to overall dynamics of the yarn.

Recently Fraser et al. have applied mathematical theory of perturbations to correctly eliminate the time dependance from equations of motion in stationary conditions (Fraser et al.,1992).

Mathematical modeling is an important tool in determining the optimal yarn motion in textile processes. Simulations make it possible to predict the dynamic properties of the yarn and the mechanical tension inside the yarn, thus it is very valuable technique in the construction of yarn packages and the textile processing equipment.

The first step in modelling is to devise a mathematical formulation of the problem. Using suitable assumptions and approximations, one tries to derive the equation of motion of the yarn that is a good description of all the relevant physical effects, but at the same time the equation must be simple enough so that it can be solved at least approximately. 


\section{Kinematics}

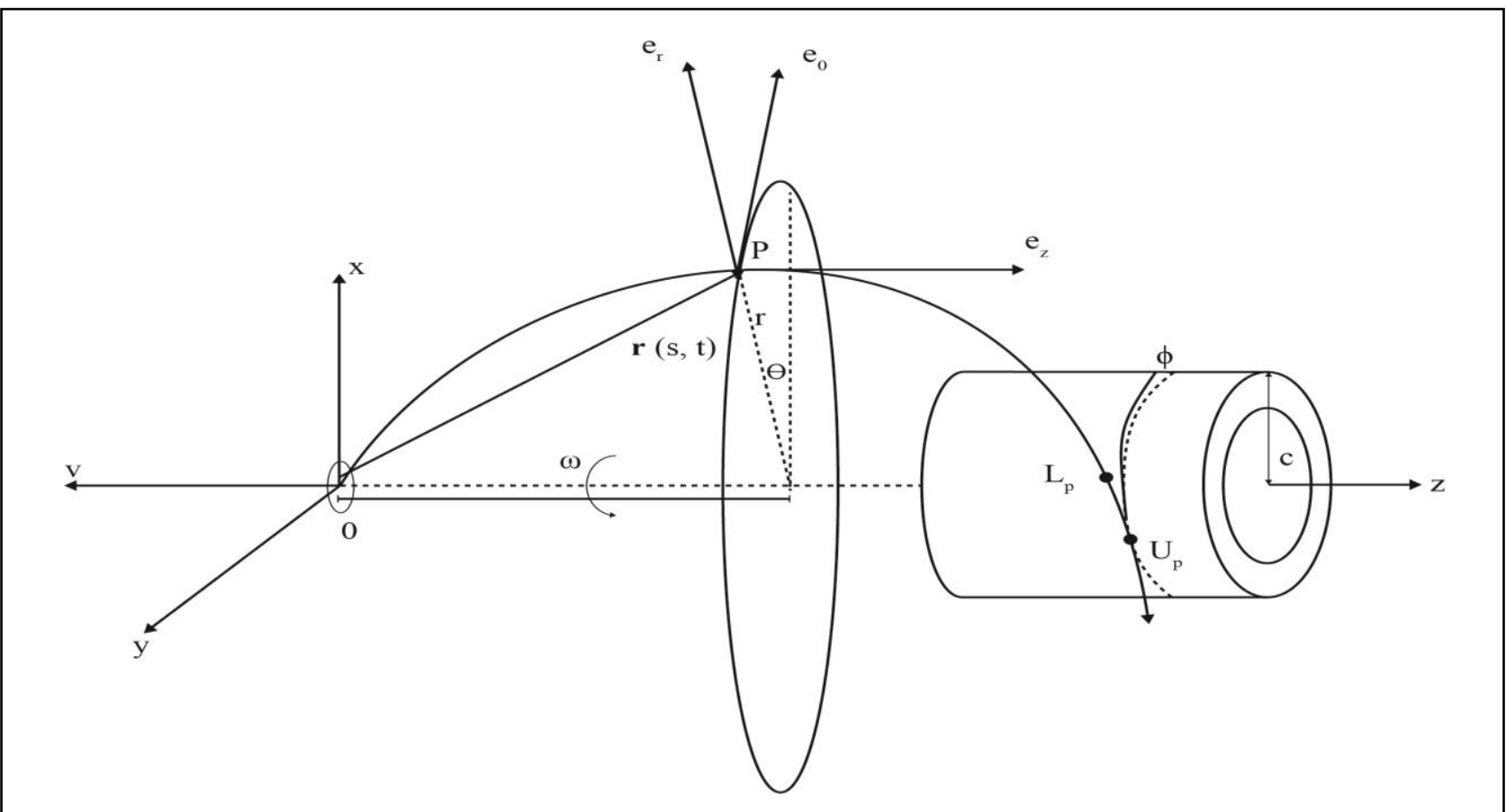

Fig. 1. Cylindrical coordinate system rotates around the $\mathrm{z}$ axis with an angular velocity $\omega$.

The yarn is parametrised with arc length $\mathrm{s}$ ( $\mathrm{s}$ is therefore the length of yarn from the origin of the coordinate system to the given point on the yarn). The coordinates of a point are given by $\mathrm{r}$, the radial distance from the axis, $\theta$, the polar angle and $\mathrm{z}$, the vertical distance from the origin (Fraser et al.,1992). It should be kept in mind that each point has its own triplet of base vectors $e_{z}, e_{\theta}, e_{r}$, respectively pointing in vertical, tangential and radial direction. The radius vector pointing to a point on the yarn can be decomposed along radial and vertical directions (the polar angle dependence is hidden in the $\mathrm{e}_{\mathrm{r}}$ vector):

$$
r(s, t)=r(s, t) e_{r}(\theta(s, t), t)+z(s, t) e_{z}
$$

We have emphasized that coordinates of a point depend explicitelyon both the time of observation $\mathrm{t}$ and on the arc length $\mathrm{s}$, where the point is located at given time t.The velocity of a point on a yarn that is being withdrawn (with withdrawing speed V) is given by the total time derivative:

$$
v=\frac{d r}{d t}=\frac{\partial r}{\partial t}+\frac{\partial r}{\partial s} \frac{\partial s}{\partial t}
$$

It is important to note that the velocity is not given by the local (partial) time derivative, denoted by $\partial \mathrm{r} / \partial \mathrm{t}$. This derivative does not take into account that in the infinitesimal time $\Delta \mathrm{t}$ the point moves to a different position along the yarn (i.e. to a different arc length $s$ ). The contribution to velocity due to this movement is described 
by the additional term $\partial \mathrm{r} / \partial \mathrm{s} \partial \mathrm{s} / \partial \mathrm{t}$. The withdrawing speed is equal to $\mathrm{V}=-\partial \mathrm{s} / \partial \mathrm{t}$ and we obtain the following expression:

$$
v=\dot{r}-V \frac{\partial r}{\partial s}
$$

where the dot denotes the partial derivative with respect to time. It's worth noting that $\mathrm{t}=\partial \mathrm{r} / \partial \mathrm{s}$ is the unit tangential vector to the yarn. Indeed the direction of the withdrawing velocity at a given point should be in the direction of the yarn.

To calculate the time derivative of the radius vector we make use of a relation between derivatives in an inertial and a rotating frame:

$$
\left(\frac{\partial}{\partial t}\right)_{K}=\left(\frac{\partial}{\partial t}\right)_{K^{\prime}}+\omega x
$$

When applied to a base vector, that is rotating around the $\mathrm{Z}$ axis together with the yarn, this equations gives

$$
\frac{\partial e_{i}(t)}{\partial t}=\omega \times e_{i}(t)
$$

The partial time derivative of the radius vector is then found to be:

$$
\dot{r}=\dot{r} e_{r}+r \dot{e}_{r}+\dot{z} e_{z}+z \dot{e}_{z}=\dot{r} e_{r}+r \theta e_{\theta}+\dot{z} e_{z}+\dot{\omega} \times\left(r e_{r}+z e_{z}\right)=v_{r e l}+\omega \times r .
$$

The final expression for the velocity of a point is of the form

$$
v=v_{r e l}+\omega \times r-V \frac{\partial r}{\partial s}
$$

The three contributions to the velocity of the point have very simple physical interpretations. The first term is the relative velocity in the non-intertial frame; it describes how the form of the yarn is changing from the point of view of an observer that is rotating together with the yarn, but it is not equal to the velocity of a given point in the non-intertial frame. (This term is dropped in the quasi-stationary approximation that we describe below.) The second term is the circular velocity of the point due to the rotation of the frame; this is the velocity of a point that is fixed in the non-inertial frame. Finally, the last term is the withdrawing velocity that we introduced above. By analogy, the acceleration of a point is given by the total time derivative of the velocity. By a lengthy but straight-forward calculation we obtain the following expression:

$$
a=a_{r e l}+2 \omega \times v_{r e l}-2 V \omega \times \frac{\partial r}{\partial s}+\omega \times(\omega \times r)+\dot{\omega} \times r-2 V \frac{\partial r e l}{\partial s}+V^{2} \frac{\partial^{2} r}{\partial^{2}}
$$


This complex expression can be given more compact form if we introduce a differential operator $\mathrm{D}$, which follows the motion of the point in the rotating frame (Fraser et al.,1992):

$$
D=\frac{\partial}{\partial t}-V \frac{\partial}{\partial s}
$$

The fact that this operator "follows the motion of the point in the rotating frame" means, that the partial time derivative operator only operates on the coordinates of the point $(r, \theta, z)$, but it gives zero when applied on the base vectors $e_{z}, e_{\theta}, e_{r}$. The simplified expression for the acceleration is

$$
a=D^{2} r+2 \omega \times(D r)+\omega \times(\omega \times r)+\dot{\omega} \times r
$$

This expression is reminiscent of an analogous expression for acceleration of a point object in a rotating frame, with partial time derivatives replaced by the differential operator D.

\section{Dynamics}

Newton's law in the form of $\mathbf{F}=$ ma, where $\mathbf{F}$ is the force on the body, a the acceleration and $\mathrm{m}$ the mass of the body, can be used to describe the motion of point bodies and the centre-of-mass motion of rigid bodies. Here we are dealing with yarn, which is a deformable body, and we want to describe not only the motion of the yarn as a whole, but also it's shape itself (Pracek, 2002).

For this reason we partition the yarn in a large number of short (infinitesimal) segments of length $\delta$ s and we apply Newton's law for each individual segment (Fig.2).

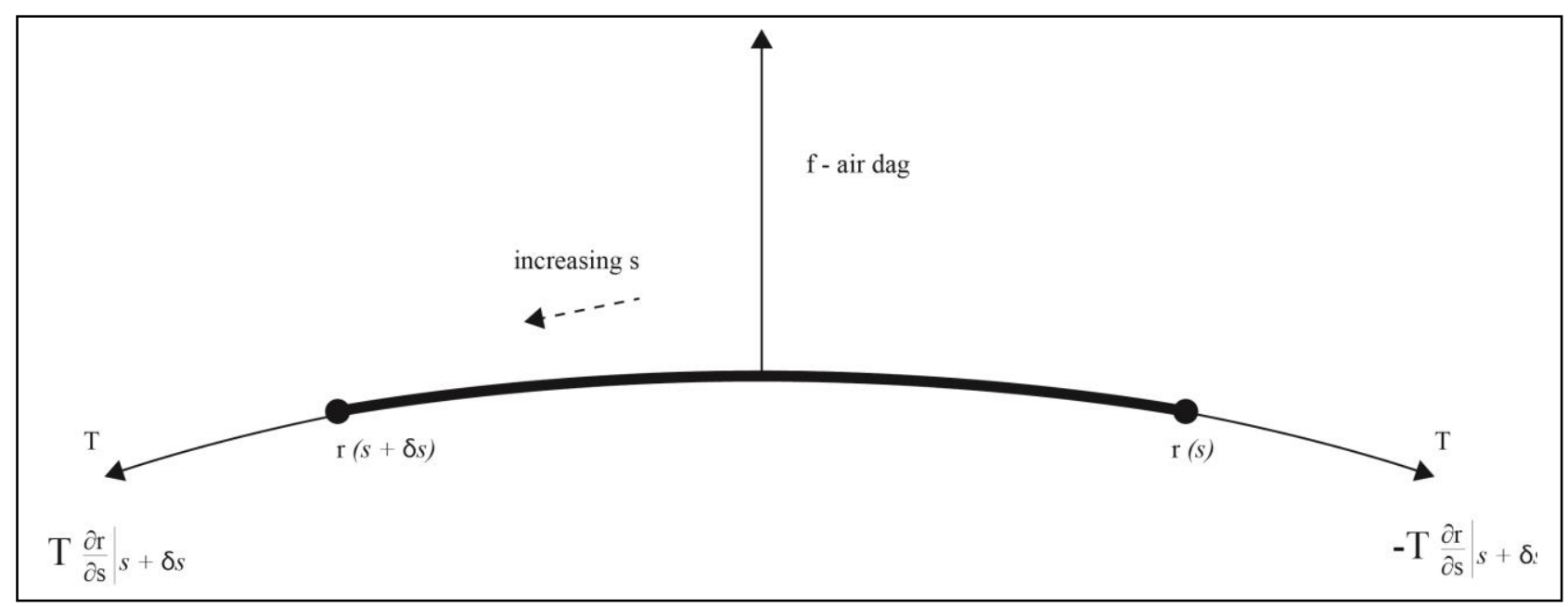

Fig. 2. A segment of yarn and forces that act on it. 
The three largest forces that act on each segment are:

- the air drag for that part of the yarn that forms the balloon (or the force of friction for the part of yarn between unwinding and lift-off point on the package, which is sliding on lower layers of yarn)

- the force imparted to the segment by the yarn "attached" to the right end point (at arclength s), - $\mathrm{T} \partial \mathrm{r} / \partial \mathrm{s}(\mathrm{s})$. Scalar $\mathrm{T}$ is the yarn tension, and the force is obviously directed along the yarn.

- the force imparted to the segmend by the yarn "attached" to the left end point (at arclength $\mathrm{s}+\partial \mathrm{s}), \mathrm{T} \partial \mathrm{r} / \partial \mathrm{s}(\mathrm{s}+\partial \mathrm{s})$.

The last two forces are due to internal elastic stress which appear because the yarn is being strained. In tridimensional bodies the elastic state is described by a tensor (stress tensor), while in a one-dimensional body such as yarn a scalar quantity $\mathrm{T}$ (tension) is sufficient. It is measured in units of force [N].

We can thus write the second Newton's law for the yarn segment as

$$
m a=\left(T \frac{\partial r}{\partial s}\right)(s+\delta s)-\left(T \frac{\partial r}{\partial s}\right)(s)+\mathbf{f}
$$

The mass of a segment is $m=\rho \delta$, where $\rho$ is the linear density of mass (i.e. mass per unit length). We write the external force $\mathbf{F}$ as $\mathbf{F}=\mathbf{f} \delta$ s, where $\mathbf{f}$ is the linear density of external force (i.e. external force per unit length). We divide the previous equation by $\delta$ s and we go the limit of infinitesimal length of the segment, $\delta s \rightarrow 0$ :

$$
\rho a=\lim _{\delta s \rightarrow 0} \frac{\left(T \frac{\partial r}{\partial s}\right)(s+\delta s)-\left(T \frac{\partial r}{\partial s}\right)(s)}{\delta s}+\mathbf{f}
$$

The limit in this expression is by definition the derivative of function $\mathrm{T} \partial \mathrm{r} / \partial \mathrm{s}$ with respect to arc-length $\mathrm{s}$. The final result, the equation of motion for an infinitesimal yarn segment, can be written as

$$
\rho a=\frac{\partial}{\partial s}\left(T \frac{\partial r}{\partial s}\right)+\mathbf{f}
$$

or, if we take into account the expression for the acceleration,

$$
\rho\left(D^{2} r+2 \omega \times D r+\omega \times(\omega \times r)+\dot{\omega} \times r\right)=\frac{\partial}{\partial s}\left(T \frac{\partial r}{\partial s}\right)+\mathbf{f}
$$

It is worthwile to cast the equation of motion in the dimensionless form. This consists of finding "natural" units in which to express all the quantities appearing in the equation. 
A number of constants will remain in the reduced equation and these dimensionless parameters have the most pronounced effect on the yarn dynamics. We express all distances in units of package radius:

$$
\bar{r}=\frac{r}{c}, \bar{z}=\frac{z}{c}, \bar{s}=\frac{s}{c}
$$

time is expressed in units of period of balloon rotation:

$$
\bar{t}=\frac{t}{\tau}=\omega t
$$

velocitis are expressed in units of unwinding speeed:

$$
\bar{v}=\frac{v}{V}, \bar{v} n=\frac{v_{n}}{V}
$$

and finally we find the following suitable combinations of quantities for forces and tension:

$$
\begin{gathered}
\mathbf{f}=\frac{f c}{\rho V^{2}} \\
\bar{n}=\frac{n c}{\rho V^{2}} \\
T=\frac{T}{\rho V^{2}}
\end{gathered}
$$

When we rewrite the equation of motion with these dimensionless quantities we obtain

$$
\bar{D}^{2} \bar{r}+2 \Omega \times D \bar{r}+\Omega \times(\Omega \times \bar{r})+\Omega \frac{\partial \Omega}{\partial t} \times \bar{r}=\frac{\partial}{\partial \bar{s}}\left(T \frac{\partial \bar{r}}{\partial \bar{s}}\right)+\mathbf{f}
$$

Only one parameter remains in this equation (without taking into account the external force term). It is the dimensionless angular velocity $\Omega$ :

$$
\Omega=\frac{c \omega}{V}
$$

This is the single most important parameter in our model. We will show later on that we can make many important conclusions if we determine how $\Omega$ changes with time as the yarn is being unwound. There are two additional dimensionless parameters: $\mu$, the coefficient of friction between yarn and package (Roberson\&Crowe, 1980), and the coefficient of air resistance $p_{0}$ (Fraser ,1992). 


$$
p_{0}=\frac{16 c D_{n}}{\rho}
$$

These two parameters are approximately constant during the unwinding, so they are less important. The coefficient of friction depends on the quality of the yarn and on the surface properties of the package. The quality of the surface is comparable for forward and backward unwinding direction, so that the coefficient of friction remains approximately constant.

\section{Simulation model}

In cylindrical packages the relation between the angular velocity of the yarn during unwinding $\omega$, the unwinding speed $V$ and the package radius $c$ at the lift-off point, where the yarn lifts off from the package surface, is (Praček et al.,2011, Praček et al.,2011):

$$
\omega=\frac{V}{c}\left(\frac{1}{\cos \phi}-\tan \phi\right)^{-1}=\frac{V}{c} \frac{\cos \phi}{1-\sin \phi}
$$

Introducing the dimensionless angular velocity $\Omega=\omega \mathrm{c} / \mathrm{V}$, this formula can also be expressed as

$$
\Omega=\frac{\cos \phi}{1-\sin \phi}
$$

We are looking for a mathematical description of the time dependence of the winding angle during the unwinding. This is of crucial importance because the dimensionless angular velocity of yarn depends on the winding angle as seen in Eq.(23), the dimensionless angular velocity has a great impact on the tension of yarn in agreement with Eg. (19). In building this model we make a few assumptions: we neglect the residual tension of yarn in the package, which is related to the stiffness of the winding, the winding angle and the number of the threads are approximately constant in the number of the layers whose unwinding will be simulated.

A function with suitable properties must be obtained from a periodic function since the movement of the point of unwinding is also approximately periodic. We therefore start with the most well-known periodic function - the sine function. We will have to suitably adjust it to reflect the slow-down of the movement close to the edges. This can be achieved by raising it to a small power, say with exponential 1/40:

$$
\mathrm{f}(t)=\left.\operatorname{sign}(\sin t) \sin t\right|^{\frac{1}{40}}
$$

To compute the 1/40-th power we had to take the absolute value of the sine function; see the second factor. The first factor, $\operatorname{sign}(\sin t)$, takes care of the right sign. The graph of function $\mathrm{f}(\mathrm{t})$ is on the figure (3) 


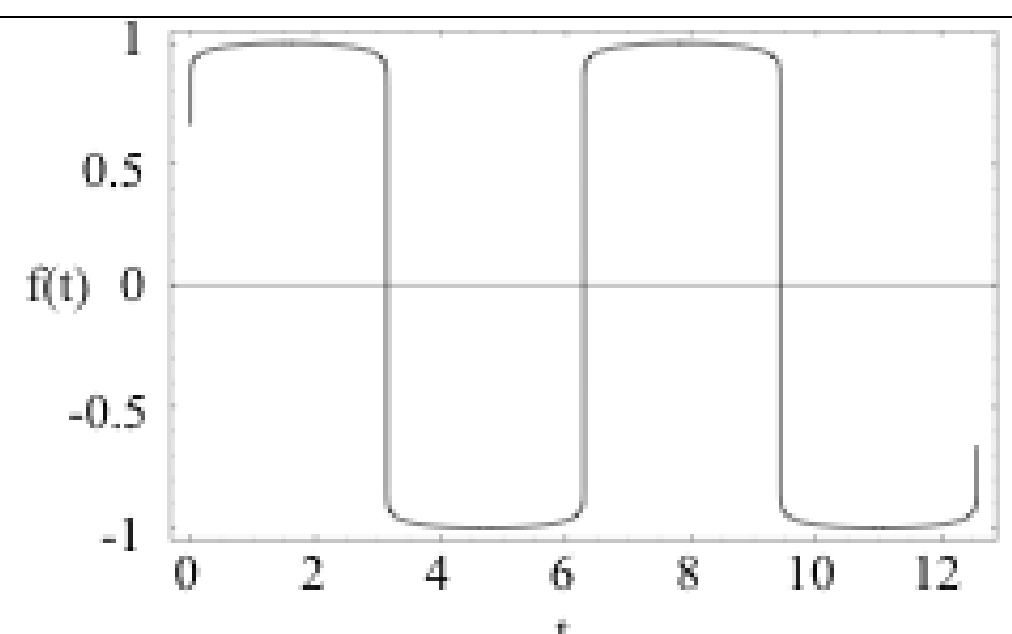

Fig. 3. Model function for winding angle.

\subsection{Results of unwinding simulation}

We have performed numerical simulation of unwinding from cylindrical package. We show the time dependence of the position of the dimensionless angular velocity. The time is expressed in units of phase: $2 \pi$ corresponds to one cycle of unwinding point up and down the package.

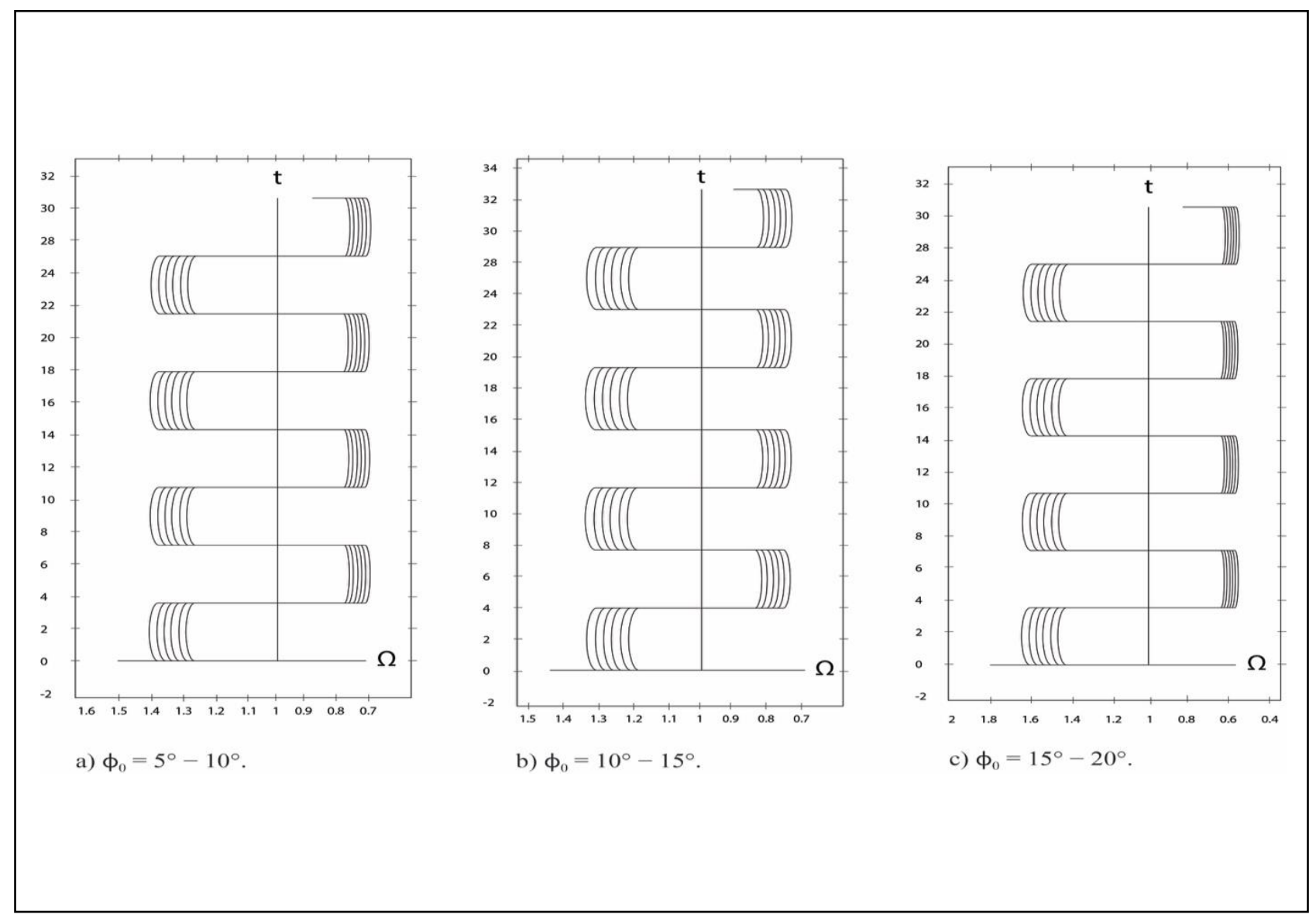

Fig. 4. Oscillations of dimensionless angular velocity $\Omega$ during the unwinding yarn from cylindrical packages, where $\phi_{0}=0^{\circ}-20^{\circ}$. 

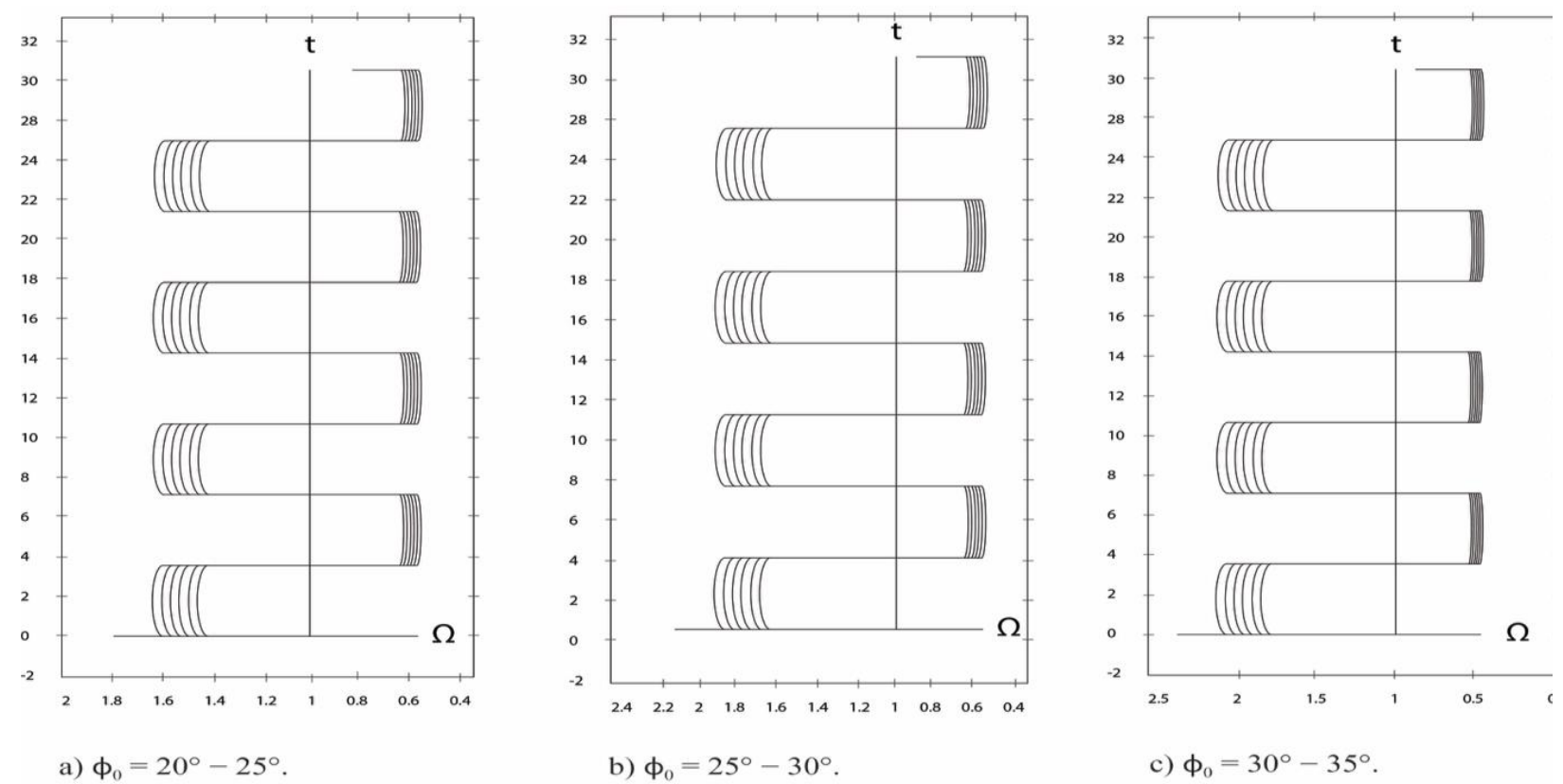

Fig. 5. Oscillations of dimensionless angular velocity $\Omega$ during the unwinding yarn from cylindrical packages, where $\phi_{0}=20^{\circ}-35^{\circ}$.

Figures 4 and 5 show the dependence of the angular velocity oscillations as a function of the winding angle from $0^{\circ}$ to $35^{\circ}$. The amplitude of dimensionless angular velocity oscillation is larger for large angular velocities and for larger winding angles.

The oscillations of yarn tension are related to the variation of the dimensionless angular velocity of yarn rotation around the package axis. The amplitude of the dimensionless angular velocity oscillations can be calculated as

$$
\Delta \Omega=\Omega_{\max }-\Omega_{\min }=\frac{\cos \phi_{0}}{1-\sin \phi_{0}}-\frac{\cos \left(-\phi_{0}\right)}{1-\sin \left(\phi_{0}\right)}=2 \tan \phi_{0} .
$$

In the region of interest, i.e. for $\phi<25^{\circ}$, we can approximate $\tan \phi \sim \phi$, so we have

$$
\Delta \Omega \approx 2 \phi_{0} .
$$

The lower the winding angle is, the lower will the oscillations of the tension be. The maxima will then be lower and we'll be able to increase the unwinding speed. The problem is that most of the winding machines in use throughout the world use circumferential driving of the tube.

This method of package winding makes it impossible to change the winding angle, while this is possible with winding machines using direct driving of the tube. In recent times the fraction of such winding machines is increasing. 


\section{Conclusion}

We have shown crucial steps in the derivation of the equation of motion of yarn: the introduction of the non-uniformly rotating obsrvation frame, the calculation of velocity and acceleration and the application of Newton's second law to an infinitesimal segment of yarn. The origin of the oscillations of yarn dimensionless angular velocity was described. We've emphasized the role of the winding angle that can have important effects near the edges of a package.

We have reached the following conclusions:

- In cross-wound packages, when the yarn unwinds backwards the angular velocity is higher as when the yarn unwinds forwards, which leads to oscillations in the dimensionless angular velocity. Abrupt changes of tension occur at the edges of the package.

- Oscillations of dimensionless angular velocity are smaller in packages with small winding angle (parallel-wound packages), but for such packages yarn slips can occur during unwinding.

- Alternatively, the oscillations of dimensionless angular velocity can be reduced if only those layers of yarn that are unwound backwards are parallely wound.

Our future research will be based on our results so far we will construct packages with a mixed layer structure, for instance packages where there is an alternation of layers with cross winding and parallel winding. The package should be designed such that the parallel layers are unwound in the backward direction, while the intermediate cross wound layers should be unwound in the forward direction. This choice is based on the observation that cross winding leads to high oscillations of yarn tension during unwinding in the backward direction. The additional benefit of cross-wound layers between two parallel layers is that the yarn in the cross-wound sections prevents the parallel layers from interleaving.

\section{References}

Barr, A. E. D., Catling, H. (1976). Manual of Cotton Spimmimg, Volume Five. Butterworth

Fraser, W. B., Ghosh, T. K., Batra, S. K. (1992). On unwinding yarn from cylindrical package. Proc. R. Soc. Lond. A, 436 479-498.

Fraser, W. B., (1992). The effect of yarn elasticity on an unwinding ballon. J. Tex. Inst, 83 603-613.

J.D.Clark, W.B.Fraser, R. Sharma and C.D.Rahn. (1998). The dynamic response of a ballooning yarn:theory and experiment. Proc. R. Soc. Lond. A, 454 2767-2789.

Kothari,V.K.,Leaf G.A.V. (1979). The unwinding of yarns from packages, PartII: The theory of yarn-unwinding.J.Text.Inst 70 (3)95-105. 
Mack, C. (1953). Theoretical study of ring and cap spinning balloon curves(with and without air drag).J.Text.Inst 44 483-498.

Padfield, D. G. (1956). The Motion and Tension of an Unwinding Thread. Proc. R. Soc., vol. A245, 382-407.

Praček, S. (2002). Modification of yarn unwinding dynamics. Dissertation, University of Ljubljana

Roberson, J. A., Crowe, C. T. (1980) Engineering fluid dynamics. Houghton Mifflin Company, Boston, second edition.

Praček, S. Sluga, F. Možina, K. Franken, G. (2011). Unwinding from cylindrical packages, Annals of DAAAM for 2011 \& Proceedings of the 22 st International DAAAM Symposium, 23-26th November 2011, Vienna, Austria, ISSN 1726-9679, ISBN 978-3-901509-83-4, Katalinic, B. (Ed), pp. 0917-0918, Published by DAAAM International Vienna, Vienna

Praček, S. Sluga, F. Možina, K. Franken, G. (2011). Cylindrical packages simulations, Annals of DAAAM for 2011 \& Proceedings of the 22 st International DAAAM Symposium, 23-26th November 2011, Vienna, Austria, ISSN 1726-9679, ISBN 978-3-901509-83-4, Katalinic, B. (Ed), pp. 0919-0920, Published by DAAAM International Vienna, Vienna 